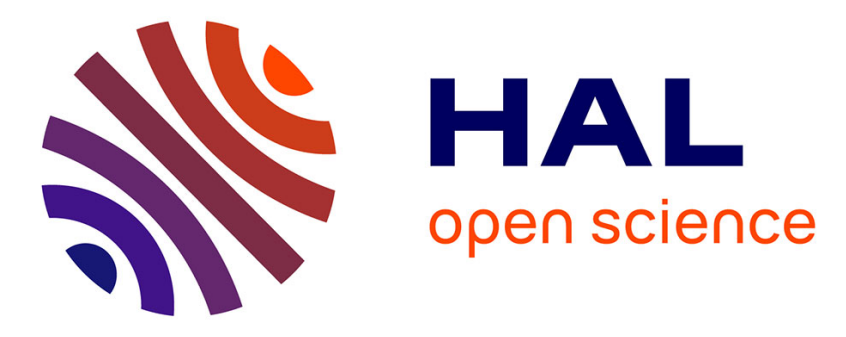

\title{
K-space data processing for Magnetic Resonance Elastography (MRE)
}

Nadège Corbin, Elodie Breton, Michel de Mathelin, Jonathan Vappou

\section{To cite this version:}

Nadège Corbin, Elodie Breton, Michel de Mathelin, Jonathan Vappou. K-space data processing for Magnetic Resonance Elastography (MRE). Magnetic Resonance Materials in Physics, Biology and Medicine, 2017, 30 (2), pp.203-213. 10.1007/s10334-016-0594-8 . hal-02385206

\section{HAL Id: hal-02385206 https://hal.science/hal-02385206}

Submitted on 28 Nov 2019

HAL is a multi-disciplinary open access archive for the deposit and dissemination of scientific research documents, whether they are published or not. The documents may come from teaching and research institutions in France or abroad, or from public or private research centers.
L'archive ouverte pluridisciplinaire HAL, est destinée au dépôt et à la diffusion de documents scientifiques de niveau recherche, publiés ou non, émanant des établissements d'enseignement et de recherche français ou étrangers, des laboratoires publics ou privés. 
K-space data processing for Magnetic Resonance Elastography (MRE)

Nadège Corbin, Elodie Breton, Michel de Mathelin and Jonathan Vappou

ICube, University of Strasbourg, CNRS, IHU Strasbourg, France

Short title: MRE using raw MR data

Address: ICube, University of Strasbourg, CNRS, IHU Strasbourg, France. ICUBE SC IRCAD, 1 place de l'hôpital, 67091 STRASBOURG CEDEX, FRANCE

Corresponding author: Jonathan Vappou, jvappou@unistra.fr, +33 388119132

Abstract: 203 words

Text: 4375 words

Number of references: 29 


\section{Abstract}

Object: Magnetic Resonance Elastography (MRE) requires substantial data processing based on phase image reconstruction, wave enhancement and inverse problem solving. The objective of this study is to propose a new, fast MRE method based on MR raw data processing, particularly adapted to applications requiring fast MRE measurement or high elastogram update rate.

Material and Methods: The proposed method allows measuring tissue elasticity directly from raw data without prior phase image reconstruction and without phase unwrapping. Experimental feasibility is assessed both in a gelatin phantom and in the liver of a porcine model in vivo. Elastograms are reconstructed with the raw MRE method and compared to those obtained using conventional MRE. In a third experiment, changes in elasticity are monitored in real-time in a gelatin phantom during its solidification by using both conventional MRE and raw MRE.

Results: The raw MRE method shows promising results by providing similar elasticity values to the ones obtained with conventional MRE methods while decreasing the number of processing steps and circumventing the delicate step of phase unwrapping. Limitations of the proposed method are the influence of the magnitude on the elastogram and the requirement for a minimum number of phase offsets.

Conclusion: This study demonstrates the feasibility of directly reconstructing elastograms from raw data.

Keywords: Elasticity Imaging Techniques; Magnetic Resonance Imaging; Radiology, Interventional 


\section{Introduction}

Magnetic Resonance Elastography (MRE) is a non-invasive technique for measuring the mechanical properties of soft tissues. MRE allows to detect elasticity changes resulting from the presence of a pathology. For example, hepatic fibrosis $[1,2]$ has been shown to be associated with increased values of shear modulus. Higher modulus values may also reflect the presence of cancerous tissue as it has been shown in liver and breast [3-5]. On the contrary, neurodegenerative disorders may be related to lower stiffness values [6-8]. MRE requires a mechanical exciter that generates a wave propagating through the tissue. The displacement resulting from the propagation of the shear wave is encoded on phase images by using a specific MR-pulse sequence. An inverse problem solver allows reconstructing an elastogram based on the fact that the shear wave velocity is directly related to the underlying mechanical properties [9].

The duration of the overall MRE exam is highly variable as it depends on the nature of the information that is searched for. Highly quantitative MRE methods aiming at measuring advanced mechanical properties such as viscoelasticity or poroelasticity $[5,10-14]$ may require highly resolved images, 3D motion encoding in several slices, advanced post-processing and inverse problem solving techniques, that will make the whole process last from several minutes up to one hour. On the other extreme, MRE for interventional radiology $[15,16]$ aims at providing the physician with an updated elastogram as fast as possible. The whole acquisition and online reconstruction process has been shown to be achievable down to a couple of seconds in interventional MRE [16]. Such fast methods cannot be considered as quantitative as biomechanically-oriented MRE methods in terms of tissue mechanical properties that are calculated. However, they allow to provide the user with a stiffness parameter at a very high refresh rate, which is highly important in specific applications such as interventional radiology. As an example of how elastography may help to interventional procedures, tissue elasticity 
has been reported as a valuable biomarker for the monitoring of thermal ablations in real-time [17, $18]$.

Fast MRE usually relies on the use of fractional encoding [19], i.e. the frequency of the motionsensitizing gradient is higher than the MRE excitation frequency, and on the use of fast MR pulse sequences such as echo-planar imaging, fast gradient echo sequence or optimal k-space filling strategies [20-22]. Despite these acceleration strategies, such methods are based on classical MRE protocol, namely, the encoding of shear wave on phase images, the reconstruction of the phase images, filtering and processing of such phase images typically with temporal Fourier transform and phase unwrapping, and the resolution of the inverse problem on the resulting data. In this paper, we propose an alternative to this process aiming at accelerating and simplifying the reconstruction of the elastogram, for applications that require fast MRE information. The key feature of the proposed method is that spatial frequencies, and hence wavelengths, are estimated directly from the raw MR data, without any need for phase image reconstruction. This allows circumventing several steps such as spatial inverse and forward Fourier transforms and unwrapping algorithms.

\section{Material and Methods}

\section{Theoretical background}

As mentioned above, conventional MRE uses MR phase data for wave image and elastogram reconstruction. The proposed method uses directly the raw complex MRI data. A schematized comparison between the two methods is provided in Fig. 1. This section introduces the theoretical background of the proposed method. 


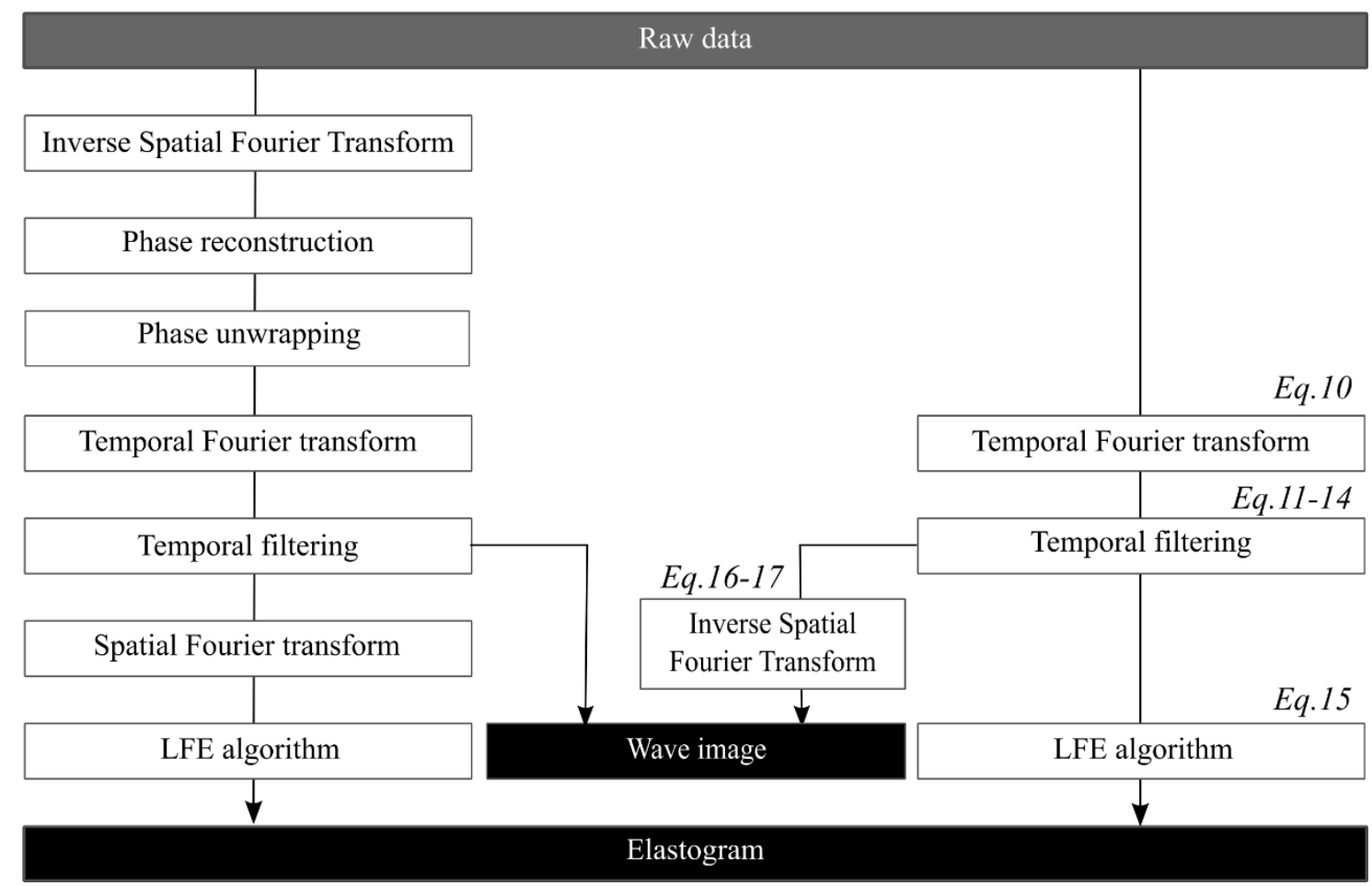

Fig. 1 Schematic description of the conventional MRE method and the proposed, raw data-based method

\section{Conventional MRE}

MRE relies on the encoding of tissue motion in the phase $\Phi$ of the complex MRI signal. Let us consider a harmonic oscillation of one isochromat with maximum amplitude $u_{0}$ at a given temporal frequency $f_{e}$, where $\vec{u}$ is the position vector:

$$
\vec{u}(t)=\overrightarrow{u_{m}}+\overrightarrow{u_{0}} \sin \left(2 \pi f_{e} t-\vec{k} \cdot \vec{u}+\alpha\right)
$$

where $\vec{k}$ is the wave number, $\overrightarrow{u_{m}}$ is the mean position and $\alpha$ is the initial phase offset.

A number $N_{p}$ of periods of a sinusoidal motion encoding gradient (MEG), called G with maximum amplitude $G_{0}$ is implemented in the MR-pulse sequence and its polarity is switched with respect to the mechanical excitation frequency $f_{e}$. 


$$
G(t)=\left\{\begin{array}{c}
G_{0} \sin \left(2 \pi f_{e} t\right) t \in\left[0, N_{p} / f_{e}\right] \\
0 \text { otherwise }
\end{array}\right.
$$

The observed MRI phase shift $\Phi_{s}$ acquired with a gradient echo sequence including the described MEG is proportional to the scalar product of the position vector and the gradient vector [9]:

$$
\Phi_{s}=\gamma \frac{N_{p}\left(\overrightarrow{G_{0}} \cdot \overrightarrow{u_{0}}\right)}{2 f_{e}} \cos (\vec{k} \cdot \vec{u}-\alpha)
$$

A similar equation can be written when the MEG frequency $f_{g}$ and the mechanical excitation frequency $f_{e}$ are not equal [19]:

$$
\Phi_{s}=\gamma \frac{f_{g} \overrightarrow{G_{0}} \cdot \overrightarrow{u_{0}}}{\pi\left(f_{g}^{2}-f_{e}^{2}\right)} \sin \left(\frac{\pi f_{e}}{f_{g}}\right) \cos \left(\frac{\pi f_{e}}{f_{g}}-\vec{k} \cdot \vec{u}+\alpha\right)
$$

More generally, the observed MRE phase shift can simply be written as:

$$
\Phi_{s}=C_{1} \cos \left(-\vec{k} \cdot \vec{u}+C_{2}\right)
$$

The same expression can be obtained for spin echo sequences with different expressions for $C_{1}$ and $C_{2}$. MRE relies on estimating the wavenumber $k$ from the phase of the acquired MRI signal. The conventional protocol involves the reconstruction of the phase image within the range $[-\pi, \pi]$, the use of a phase unwrapping algorithm, the harmonic analysis on a set of images with different initial phase offsets $\alpha$, and finally, the resolution of the inverse problem.

The objective of this study is to propose a new approach that circumvents several of the aforementioned steps while being more adapted to fast MRE protocols. 
MRE using raw data

The proposed theoretical framework will be developed in 1D for clarity. It can be easily extended to 2D images. The raw MRI signal $S$ acquired in the k-space can be defined with respect to the transverse magnetization $I(x)$ as:

$$
S\left(v_{x}\right)=\sum_{x=0}^{N_{x}-1} I(x) e^{-j 2 \pi \frac{v_{x} x}{N_{x}}}
$$

where $x$ is the arbitrary discrete spatial dimension considered in this example, $N_{x}$ is the total number of points, $v_{x}$ is the discrete spatial frequency and $I(x)$ is the transverse magnetization $I(x)=$ $M(x) e^{j \Phi(x)}, M$ being its magnitude and $\Phi$ being its phase. In the specific case of MRE, $\Phi$ is given by:

$$
\Phi(x)=\Phi_{0}(x)+\Phi_{s}(x)
$$

where $\Phi_{0}$ is the background phase, i.e. the constant phase obtained without motion encoding, and $\Phi_{S}$ is the phase shift related to the encoded displacement as described in Eq.[5].

The k-space acquired in MRE experiments can therefore be written as follows:

$$
S\left(v_{x}\right)=\sum_{x=0}^{N_{x}-1} M(x) e^{j \Phi_{0}(x)+j C_{1}(x) \cos \left(-\frac{k_{x} x}{N_{x}}+C_{2}\right)} e^{-\frac{j 2 \pi v_{x} x}{N_{x}}}
$$

where $\frac{k_{x}}{N_{x}}$ corresponds to the normalized discrete wave number.

Eq.8 includes an exponential of a cosine function that can be rewritten using the Jacobi-Anger expansion [23]

$$
S\left(v_{x}\right)=\sum_{x=0}^{N_{x}-1} M(x) e^{j \Phi_{0}(x)} e^{-\frac{j 2 \pi v_{x} x}{N_{x}}} \sum_{n=-\infty}^{\infty} j^{n} J_{n}\left(C_{1}(x)\right) e^{-\frac{n k_{x} x}{N_{x}}+n C_{2}}
$$


where $J_{n}$ is the $\mathrm{n}^{\text {th }}$ Bessel function. This equation highlights the presence of an infinite number of harmonics of the spatial frequency of interest in the k-space due to the presence of a cosine pattern in the phase signal. A similar characteristic has already been described in the framework of motion compensation in MRI [24].

In order to illustrate this phenomenon, a simulated 1D MRE signal is represented in Fig. $\mathbf{2}$ showing the simulated spectrum of the resulting MRE 1D-signal. In this figure and the following, only the modulus of complex signals are represented in the frequency domain.

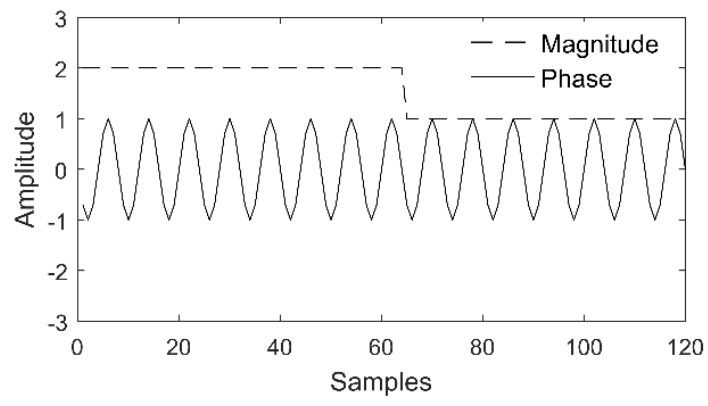

a

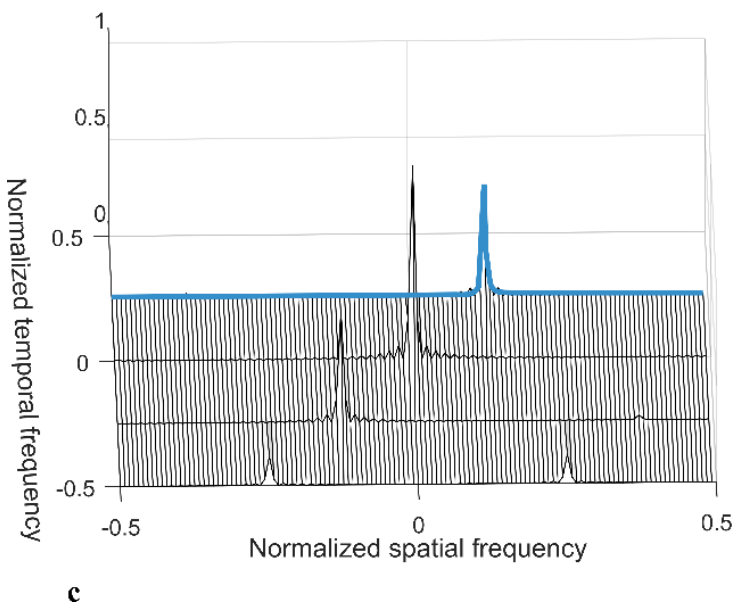

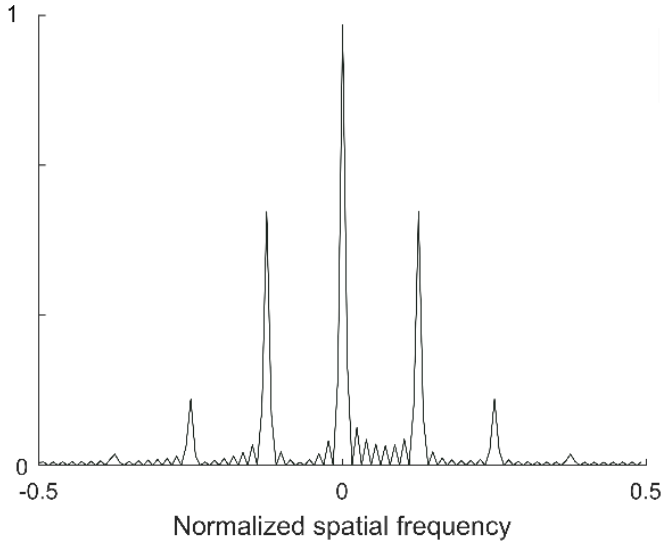

b

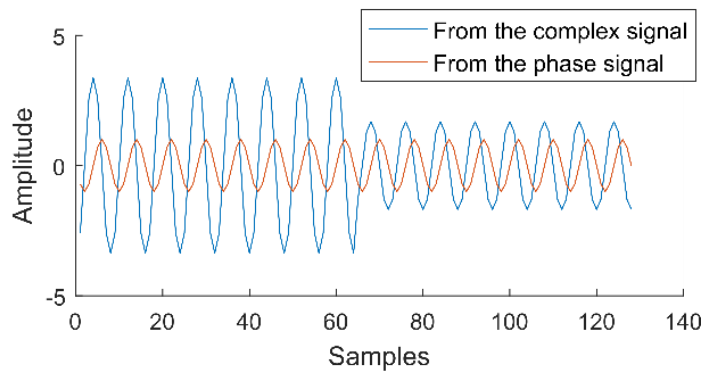

d

Fig. 2 a) Simulated 1D MR signal with heterogeneous magnitude and sinusoidal phase. b) Raw data corresponding to the simulated 1D MR signal. c) Plot of the temporal Fourier transform of a simulated data set 
with 4 varying delays between excitation and MEG. Selection of the component of interest of the spatial frequency in blue. d) Wave signal obtained from the phase signal in red (conventional process) and wave signal obtained from the complex signal in blue (new proposed method). All ordinates are in arbitrary units

The spectrum is composed of an infinite number of harmonics as predicted by Eq.[9]. In order to remove all harmonics and select only the component of interest, a simple low pass filter could be used. However, the intrinsic heterogeneity of real tissue results in the presence of several spatial frequencies of interest (Fig. 3). Hence, distinguishing the component of interest of one frequency from the harmonic of another one is impossible.

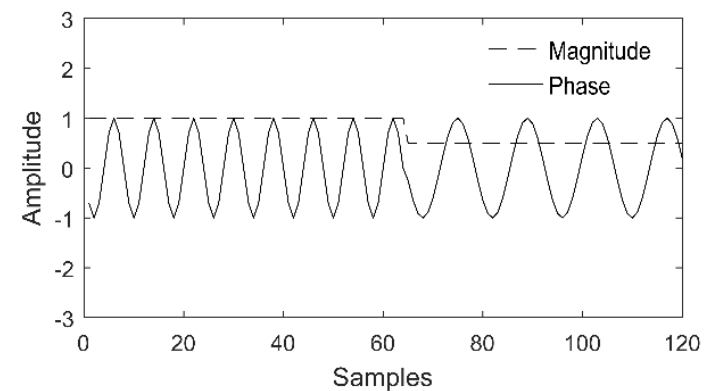

a

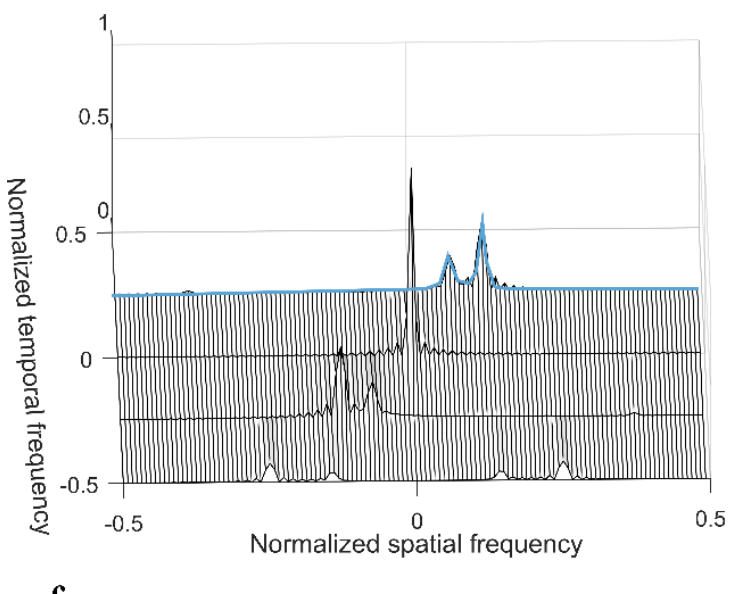

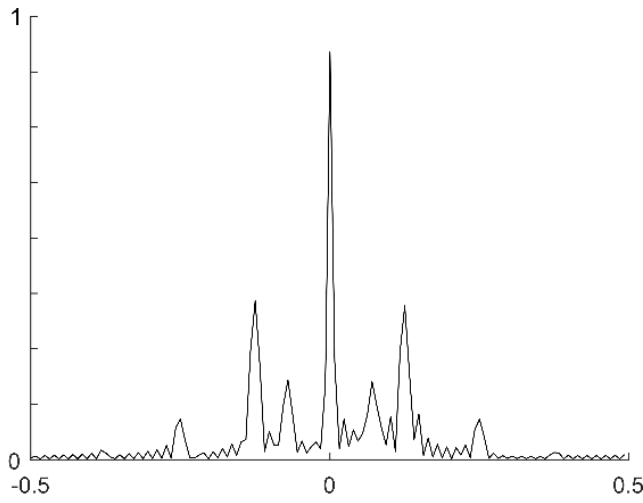

b

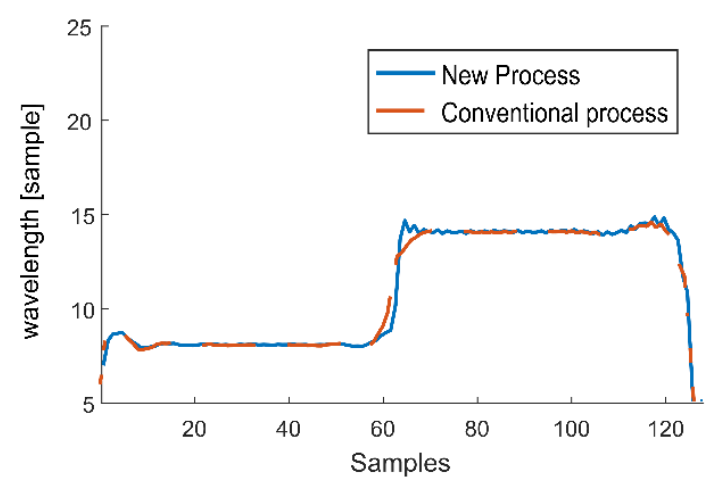

d

Fig. 3 a) Simulated 1D MR signal with heterogeneous magnitude and a signal composed of two frequencies. b) Raw data corresponding to the simulated 1D MR signal. c) Plot of the temporal Fourier transform of a simulated data set with 4 varying delays between excitation and MEG. Selection of the component of interest of the spatial 
frequency in blue. d) Local frequencies returned by the LFE-algorithm performed on the filtered k-space (blue) compared to the conventional result obtained with the same filters (red) applied to phase signal

The proposed solution is inspired from the harmonic analysis used in conventional MRE. As previously mentioned, several images are acquired with varying phase shifts between mechanical excitation and motion encoding gradients. A temporal Fourier transform is subsequently performed on phase images in order to select only the displacement induced by the generated mechanical wave at a specific excitation frequency. The following paragraph aims at demonstrating that a similar analysis directly performed on the raw data allows identifying and extracting the components of interest.

Let us consider $N_{t}$ acquisitions with varying phase offsets evenly spaced around the mechanical excitation cycle (i.e. sampling frequency $f_{s}$ equal to $N_{t} f_{e}$ ). The temporal discrete Fourier transform of the series, including the Jacobi-Anger expansion yields:

$$
\begin{aligned}
& S\left(v_{x}, v_{t}\right) \\
& =\sum_{x=0}^{N_{x}-1} M(x) e^{j \Phi_{0}(x)} e^{-\frac{j 2 \pi\left(v_{x} x\right)}{N_{x}}} \sum_{n=-\infty}^{\infty} j^{n} J_{n}\left(C_{1}(x)\right) e^{-j n \frac{\left(k_{x} x\right)}{N_{x}}+j n C_{2}} \sum_{t=0}^{N_{t}-1} e^{-\frac{j 2 \pi\left(v_{t} t\right)}{N_{t}}} e^{\frac{j n(2 \pi t)}{N_{t}}}
\end{aligned}
$$

Figure 2.c illustrates the effects of the temporal Fourier transform on the previously simulated signal with 4 varying phase-offsets evenly spaced around the mechanical excitation. Spatial harmonics are thus separated over the temporal frequency range. The spatial component of interest is now distinguishable since it is associated to the temporal frequency of interest.

Let us consider the previous expression Eq.[10] at the temporal excitation frequency $f_{e}$ (with $f_{e}=\frac{f_{s}}{N_{t}}$ which corresponds to $\left.v_{t}=1\right)$ : 


$$
\begin{aligned}
& S\left(v_{x}, 1\right) \\
& =\sum_{x=0}^{N_{x}-1} M(x) e^{j \Phi_{0}(x)} e^{-\frac{j 2 \pi\left(v_{x} x\right)}{N_{x}}} \sum_{n=-\infty}^{\infty} j^{n} J_{n}\left(C_{1}(x)\right) e^{-j n \frac{\left(k_{x} x\right)}{N_{x}}+j n C_{2}} \sum_{t=0}^{N_{t}-1} e^{-j 2 \pi \frac{t}{N_{t}}} e^{j n 2 \pi \frac{t}{N_{t}}}
\end{aligned}
$$

The sum over $N_{t}$ is non null and equal to $N_{t}$ only if $n=1+k N_{t}\left(k \in Z^{*}\right)$, therefore the Eq.[11] reduces to:

$$
\begin{gathered}
S\left(v_{x}, 1\right)=\sum_{n \in U} \sum_{x=0}^{N_{x}-1} M(x) e^{j \phi_{0}(x)} e^{-\frac{j 2 \pi\left(v_{x} x\right)}{N_{x}}} j^{n} N_{t} J_{n}\left(C_{1}(x)\right) e^{j n\left(-\frac{\left(k_{x} x\right)}{N_{x}}+C_{2}\right)} \\
U=\left\{n \mid n=k N_{t}+1, k \in Z^{*}\right\}
\end{gathered}
$$

Let us assume that the number of phase-offsets is sufficient to satisfy the following condition:

$$
\log \left(\frac{\left|J_{1}\left(C_{1}(x)\right)\right|}{\left|J_{k N+1}\left(C_{1}(x)\right)\right|}\right)>1, k \in Z^{*}
$$

Details of this condition are discussed in Appendix A. Eq.[12] then simplifies to:

$$
S\left(v_{x}, 1\right)=\sum_{x=0}^{N_{x}-1} M(x) e^{j \phi_{0}(x)} e^{-\frac{j 2 \pi\left(v_{x} x\right)}{N_{x}}} j N_{t} J_{1}\left(C_{1}(x)\right) e^{j\left(-\frac{k_{x} x}{N_{x}}+C_{2}\right)}
$$

The component of interest is separated from the harmonics and selected (blue line in Fig. 2). The data associated to Eq.[14] will be referred to as the filtered k-space raw data, since only the spatial frequencies associated to the temporal frequency of interest are selected.

At this point, one of the interesting features of the proposed method is that it offers the possibility of estimating the wavelength distribution using the Local Frequency Estimation (LFE) algorithm. The LFE algorithm is a commonly used method for the resolution of the inverse problem in MRE. In conventional MRE with LFE processing, post-processed phase images are spatially Fourier transformed and filtered in order to find spatial distribution of spatial frequencies [25]. With our method, the LFE 
calculations can be directly performed on the filtered k-space data (Eq. [14]), circumventing the need for an additional spatial Fourier transform step. For this purpose, classic image filters of the LFE algorithm [26] conventionally used in the frequency domain of the phase signal, are applied to the filtered k-space raw data. The local frequency of a real signal is defined as the derivative of the phase of its analytic signal. A pair of two lognormal quadrature filters $R_{1}$ and $R_{2}$ are classically applied in the frequency domain of the enhanced wave image and the local frequency $f_{l}$ is given by:

$$
f_{l}=\sqrt{f_{1} f_{2}} \operatorname{Re}\left(\frac{\mathrm{F}^{-1}\left(\mathrm{R}_{1} \mathrm{~S}\right)}{\mathrm{F}^{-1}\left(\mathrm{R}_{2} \mathrm{~S}\right)}\right)
$$

where $f_{1}$ and $f_{2}$ are the central frequencies of $R_{1}$ and $R_{2}$, respectively. $F^{-1}$ is the inverse Fourier transform. Usually, $S$ is the signal in the spatial frequency domain of the enhanced wave image resulting from phase reconstruction, phase unwrapping, temporal frequency filtering and finally spatial Fourier transform. In this work, it is proposed to apply the same algorithm on k-space data obtained after temporal frequency filtering. In this case, $S$ is described by Eq.[16]. This complete process is illustrated in Fig. 3 on a 1D simulated MRI signal, yielding the local frequency for each sample. This method allows to reconstruct LFE-derived elastograms without phase image reconstruction and without spatial Fourier transform since LFE filtering is directly performed in the kspace. It is important to note that this method circumvents the problem of the phase wrapping, since the k-space of a signal with a phase wrapped in the range $[-\pi ; \pi]$ and the same theoretical signal with a continuous phase out of the range $[-\pi ; \pi]$ are exactly the same. The proposed method processes directly data in the k-space (Eq. [14]) and is therefore not affected by phase wrapping as would conventional phase images be. It must be noticed that the filtered k-space raw data is not the same as the k-space of the phase signal alone. Firstly, the filtered k-space raw data does not include high spatial frequencies related to the steep variations occurring in a wrapped phase. Secondly, the filtered k-space is convolved by the magnitude spectrum. 
A qualitative visualization of the shear wave pattern is possible by performing an inverse spatial Fourier transform of the filtered k-space data, yielding the following equation:

$$
s(x)=\frac{N_{t}}{N_{x}} M(x) e^{j \phi_{0}} j J_{1}\left(C_{1}(\mathrm{x})\right) \mathrm{e}^{\mathrm{j}\left(-\frac{\mathrm{k}_{\mathrm{x}} \mathrm{x}}{\mathrm{N}_{\mathrm{x}}}+\mathrm{C}_{2}\right)}
$$

The imaginary part of Eq. [16] is equal to:

$$
\left.\operatorname{Im}(s(x))=\frac{N_{t}}{N_{x}} M(x) J_{1}\left(C_{1}(\mathrm{x})\right) \cos \left(-\frac{\mathrm{k}_{\mathrm{x}} \mathrm{x}}{\mathrm{N}_{\mathrm{x}}}+\mathrm{C}_{2}+\Phi_{0}\right)\right)
$$

It must be highlighted that the wave pattern described by Eq.[17] is weighted by the magnitude $M(x)$ and Bessel coefficients that depend on the encoded wave amplitude $C_{1}(\mathrm{x})$. Besides, a phase shift related to the background phase is introduced. The relationship between this signal and the actual displacement is therefore highly complex. This signal cannot be directly compared to the displacement image usually obtained in conventional MRE, and it is not directly exploitable for further processing. However, Eq. [17] allows obtaining a qualitative visualisation of the shear wave propagation without any need for phase image reconstruction.

\section{Experimental Methods}

\section{Phantom experiments}

The proposed method was evaluated on a gelatin phantom $(17 \mathrm{~cm}$ in diameter) made of two parts with different concentrations (4\% and $8 \%$ ). A commercial pneumatic surface exciter (Resoundant, Rochester, MN) was placed on the top of the phantom in order to generate shear waves. The experiment was performed in a 1.5 T MRI scanner (MAGNETOM Aera, Siemens, Germany) and bipolar motion encoding gradients were implemented in a spoiled gradient echo sequence. The body antenna was used to obtain a single channel data set. The excitation and the encoding frequencies were set to $120 \mathrm{~Hz}$ and $220 \mathrm{~Hz}$, respectively. The motion encoding direction was set through slice and the slice 
was parallel to the surface of the pneumatic exciter. 4 phase-offsets evenly spaced across a mechanical period were acquired. Main imaging parameters are the following: FOV $340 \mathrm{~mm} \times 340 \mathrm{~mm}$, acquisition matrix $128 \times 128$, slice thickness $10 \mathrm{~mm}$, MEG amplitude $20 \mathrm{mT} / \mathrm{m}$, TE/TR 6.13/8.33 ms, flip angle $15^{\circ}$ and bandwidth frequency $795 \mathrm{~Hz} /$ pixel. The proposed raw MRE method is used to reconstruct the wave pattern image and the elastogram, both directly from the raw data without reconstructing nor the phase nor the amplitude image. These results are compared to those obtained with conventional MRE involving phase image reconstruction, followed by the use of an unwrapping algorithm [27] and temporal Fourier transform. For this experiment and the two following ones, the LFE algorithm is applied with 10 pairs of filters in 4 orthogonal directions. The smaller central frequency of the filters is 0.07 cycle/sample.

\section{In vivo experiments}

In vivo experiments were performed on swine liver and were approved by the local ethics committee (ICOMETH C2EA - 38). The animal was anesthetized (propofol $3 \mathrm{mg} \mathrm{kg}^{-1}$, pancuronium $0.2 \mathrm{mg} \mathrm{kg}^{-1}$ and isoflurane $2 \%$ ) and placed in the large bore $1.5 \mathrm{~T}$ scanner. The body antenna was used. A surface pneumatic exciter was strapped around the chest and one slice perpendicular to the surface exciter was acquired. Relevant parameters of acquisition include: excitation/encoding frequencies $40 / 90 \mathrm{~Hz}$, encoding direction: through slice, slice thickness $10 \mathrm{~mm}$, FOV $320 \mathrm{~mm} \times 320 \mathrm{~mm}$, acquisition matrix $128 \times 128$, MEG amplitude $20 \mathrm{mT} / \mathrm{m}$, TE/TR $12.7 / 25 \mathrm{~ms}$, flip angle $15^{\circ}$ and bandwidth frequency 795 $\mathrm{Hz} /$ pixel. 4 images with different phase-offsets evenly spaced across a mechanical period were acquired. The raw MRE method was compared to the conventional MRE in the same manner as in the phantom experiment. Images were acquired in a single end expiration breath-hold.

Real-time monitoring of elasticity changes in phantom

A third experiment was performed on a phantom in order to evaluate the possibility of monitoring elasticity changes without reconstructing any phase image. This experiment is similar to the experiment described in [16]. Shortly, a hollow gelatine phantom (10\%) was filled with freshly 
prepared (still liquid) gelatine (15\%) (Fig. 4). The elasticity changes occurring during the solidification of the gelatine were observed over time thanks to continuous acquisition of MRE data. MRE experiments were performed during the first 50 minutes of the cross-liking process of gelatin. During these first 50 minutes, the inclusion is still softer than the matrix even though its concentration in gelatin is higher.

A needle MRE driver was used to generate the mechanical wave. Relevant parameters of MRE acquisition include: excitation/encoding frequency: 120/290 Hz, MEG amplitude $20 \mathrm{mT} / \mathrm{m}$, encoding direction: through slice, one slice orthogonal to the needle, slice thickness $10 \mathrm{~mm}$, FOV $300 \mathrm{~mm} \times 300$ $\mathrm{mm}$, acquisition matrix $128 \times 128, \mathrm{TR} / \mathrm{TE} 8.34 / 5.62 \mathrm{~ms}$, flip angle $15^{\circ}$ and bandwidth frequency 780 $\mathrm{Hz} /$ pixel. 3 phase offsets were acquired. The $12 \mathrm{~cm}$ loop coil was placed around the needle MRE driver and used for data acquisition.

The proposed method and the conventional method were compared for estimating the variation of elasticity in the region of interest. In the conventional MRE method, phase images are reconstructed, unwrapped, and submitted to a temporal Fourier transform. Finally, only one frequency is selected and the LFE is applied to the resulting images (Fig. 4.b). In the proposed method, elastograms are reconstructed as described in the theoretical part, without reconstructing any phase image. The temporal Fourier transform is directly applied on the k-space raw data (Fig. 4.c). The LFE is applied on the resulting filtered data (Fig. 4.d). The elasticity in the same region of interest is calculated as a function of time. 

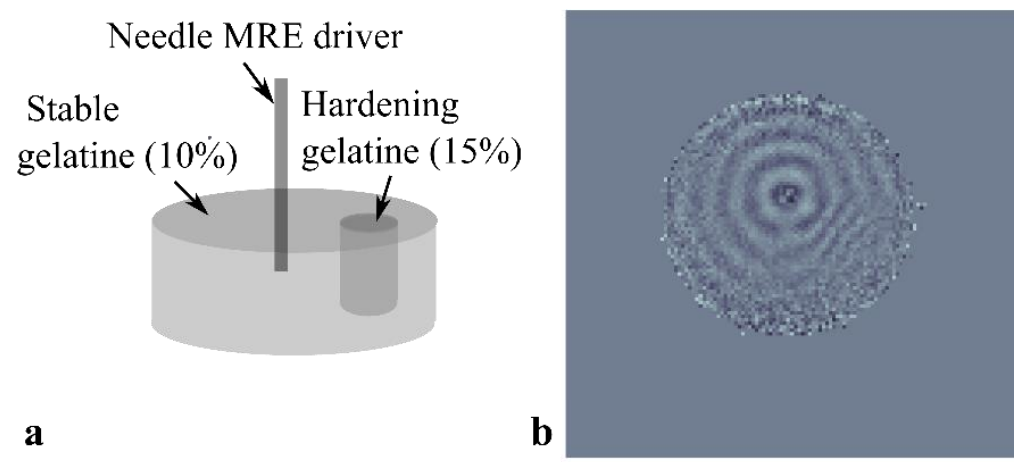

a

b
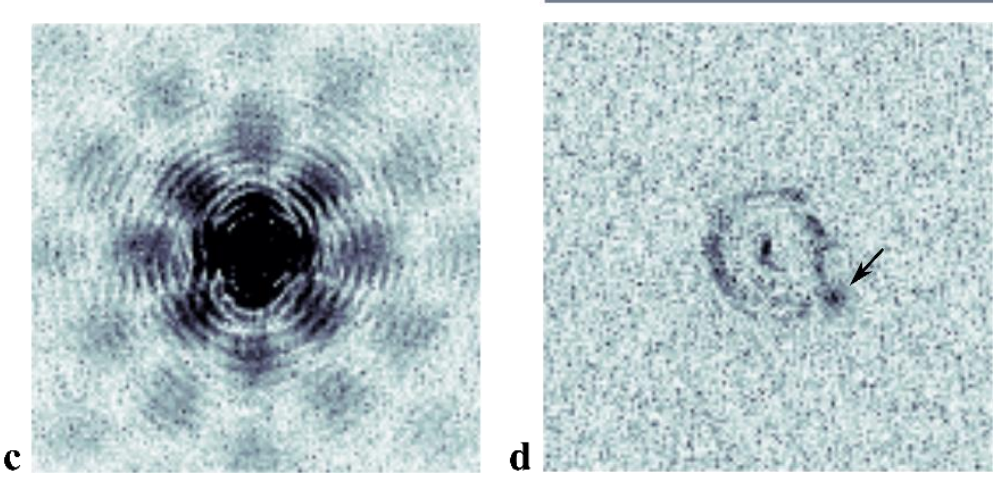

Fig. 4 a) The needle MRE driver is inserted in a stable gelatine phantom (10\%). A small part of the phantom is composed of gelatine (15\%) still under solidification process. b) Conventional phase image after phase unwrapping and temporal filtering on resulting phase images. c) Magnitude of the k-space raw data. d) Magnitude of the filtered k-space raw data at $t=1280 \mathrm{~s}$. The ring corresponds to the frequency of the wave propagating in the stable gelatin, the small region indicated by the arrow corresponds to the wave propagating in the hardening gelatin.

\section{Results}

Fig. 5 illustrates the results obtained from a conventional MRE processing including phase unwrapping (left column) and the proposed method without phase image reconstruction (right column). A qualitative shear wave pattern obtained using Eq. [17] is displayed (Fig. 5.b) for visual comparison with a displacement image obtained by conventional MRE (Fig. 5.a). LFE-derived elastograms obtained by both methods are very similar (Fig. 5.c-d). Mean values and standard deviations of the local wavelength estimation obtained with the raw MRE method and the conventional method are 
respectively $20.9 \pm 10.9 \mathrm{~mm}$ and $19.81 \pm 10.7 \mathrm{~mm}$ in the left softer half, and $30.7 \pm 4.8 \mathrm{~mm}$ and 32.7 $\pm 3.8 \mathrm{~mm}$ in the right stiffer half.
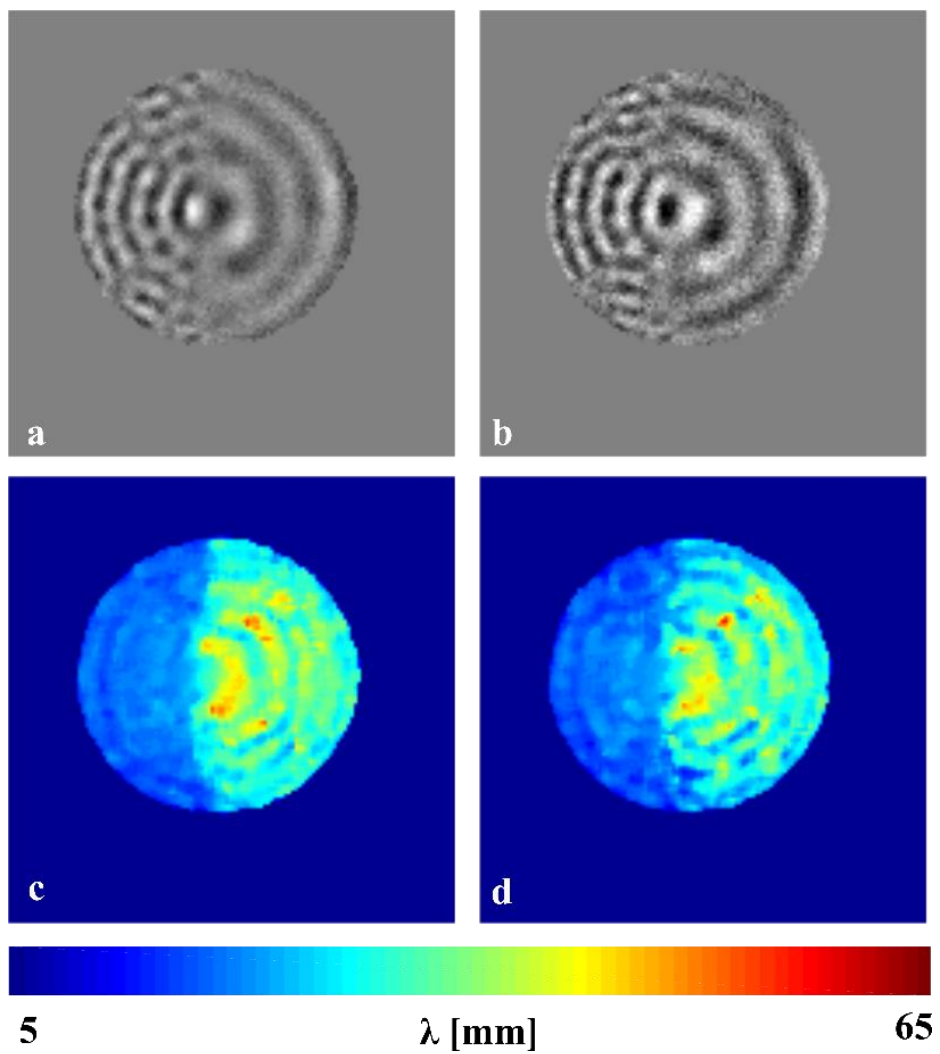

Fig. 5 (At the top) (a) Wave image obtained by unwrapping the phase image (conventional method) compared to the wave pattern image returned by the raw MRE method (b). (At the bottom) (c) Elastogram reconstructed by the conventional method compared to the one returned by the raw MRE method (d). A median filter (kernel $4 \times 4)$ was applied on the 2 resulting elastograms

Wave pattern images and LFE-derived elastograms obtained in vivo by the two methods are displayed in Fig. 6. The local wavelength obtained by the raw MRE method and conventional MRE in the liver is equal to $28.45 \pm 5.44 \mathrm{~mm}$ and $30.50 \pm 7.9 \mathrm{~mm}$, respectively. In both phantom and in vivo experiments, mean values and standard deviations of the local wavelength obtained with the proposed method were found to be very close to those obtained with the conventional one. 

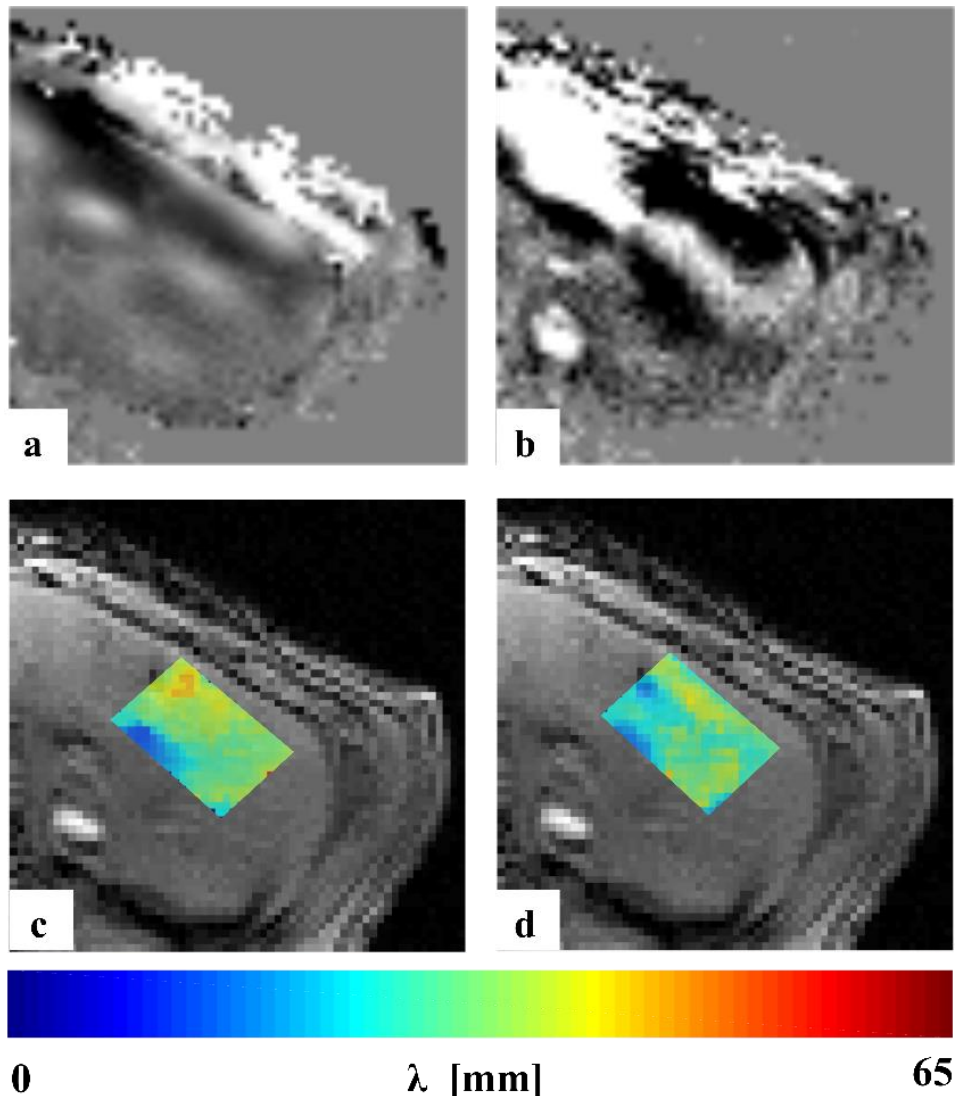

Fig. 6 (At the top) (a) Wave image obtained by unwrapping the phase image (conventional method) compared to the wave pattern image returned by the raw MRE method (b). (At the bottom) (c) Elastogram reconstructed by the conventional method compared to elastogram returned by the raw MRE method (d). A median filter (kernel 4x4) was applied on the two resulting elastograms

The variation of elasticity observed during the solidification of the gelatin is plotted in Fig. 7. Elasticity is estimated in real-time without phase image reconstruction and compared to the elasticity measured by the conventional MRE method. The evolution of the shear modulus over time obtained with the proposed method (red curve) is very similar to the one obtained with the conventional MRE method (blue curve). 


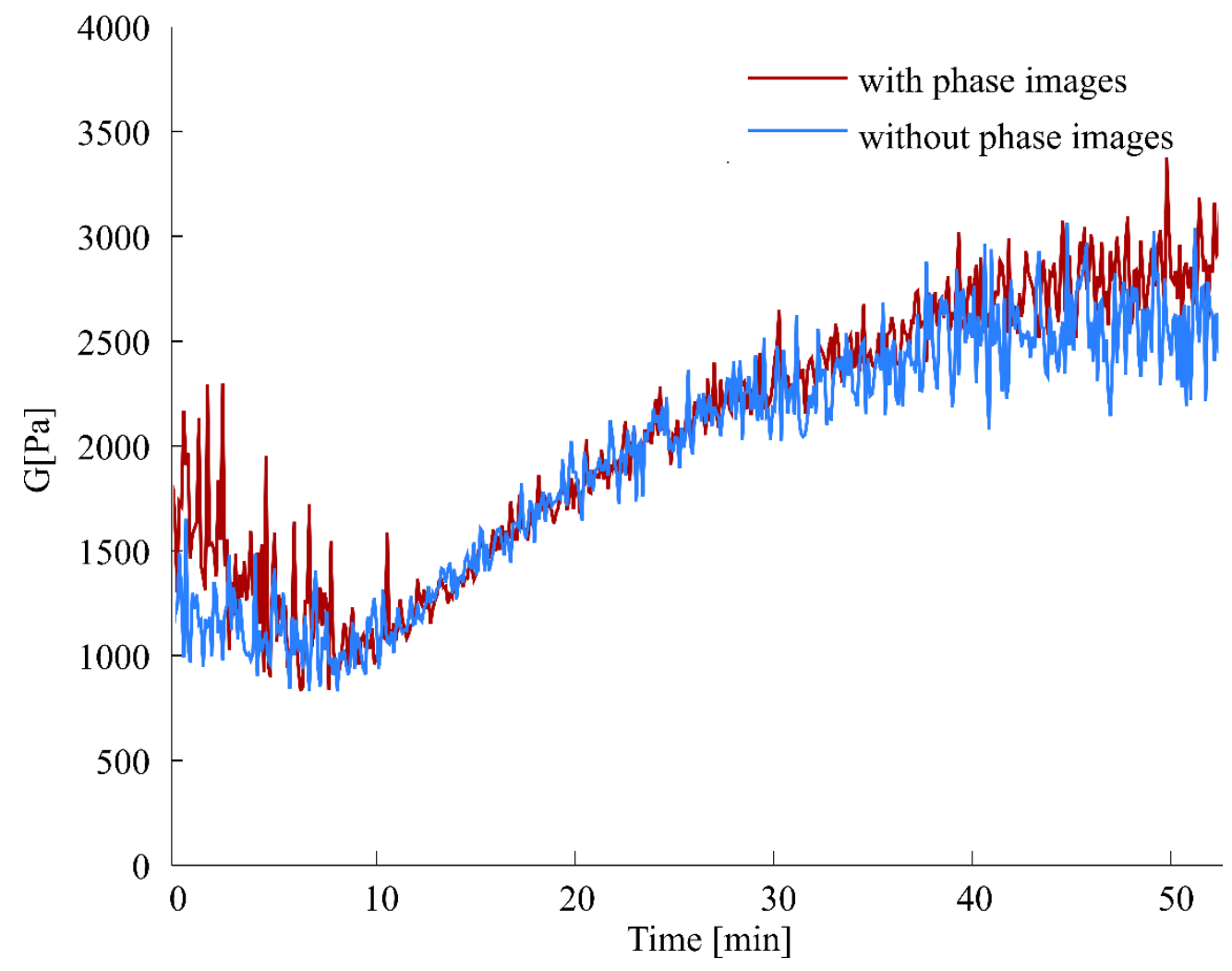

Fig. 7 Evolution of shear elastic modulus of the gelatin during its solidification measured on elastograms reconstructed by the conventional MRE (red), and the proposed raw data-based method (blue)

\section{Discussion}

This study introduces an alternative to the conventional processing protocol used in MRE. Elastograms can be reconstructed using directly raw MRI data, without phase reconstruction, phase unwrapping and spatial 2D transforms, as summarized in Fig. 1. This simplified and accelerated process is particularly interesting for applications that require very fast elastogram reconstruction or those requiring high elastogram update rate, such as in interventional radiology. The raw data obtained during an MRE acquisition is not easily readable because of the infinite number of harmonics induced by the presence of one sinusoidal pattern in the phase signal. The solution proposed here to overcome this difficulty is to perform the harmonic analysis directly on raw data. The method was assessed experimentally in gelatin phantoms and in vivo, and results were found to be very similar to those obtained with the conventional MRE process with phase unwrapping. The potential of the proposed 
method for providing elasticity changes at high temporal sampling rate was demonstrated in a third experiment where elasticity changes were monitored in gelatin during its solidification.

Fundamental aspects of the proposed method need to be discussed. As previously highlighted, this method is sensitive to the choice of the number of phase-offsets. This number must be high enough to allow the selection of only one frequency component. However, this method is intended to applications requiring fast MRE information, which assumes the use of particularly fast MRE sequences. In most of the cases, such sequences involve lower amplitude encoding than conventional MRE sequences due to acceleration strategies such as fractional encoding. This makes the condition outlined in Appendix A easier to reach. The second point that deserves to be mentioned is the assumption concerning the phase $\phi_{0}$ accumulated without the motion encoding gradient. The present theory relies on the assumption that $\phi_{0}$ is locally uniform. Should this assumption not be valid, the phase offset could be removed similarly to what is commonly done in conventional MRE, i.e. by performing a difference between two images acquired with opposite MEG polarities. However, in this case, the phase difference could then be performed through a direct product in the spatial complex domain or a convolution in the frequency complex domain, the former avoiding the need for an additional direct/inverse spatial Fourier transform. One of the main advantages of the proposed method is that it is inherently immune to phase wrapping, a known challenge in $\operatorname{MRE}[28,29]$ because its framework remains in the complex domain. Indeed, a complex signal with a theoretical phase signal out of the range $[-\pi ; \pi]$ and the same signal with a phase wrapped in the range $[-\pi ; \pi]$ have exactly the same spectrum.

The major limitation of the reconstruction of elastograms without phase images is that it is limited to the use of a unique inverse problem solving approach, namely, the LFE method. This algorithm is particularly well adapted for the proposed method, as it operates on k-space data usually obtained through spatial Fourier transform of filtered phase images. With the proposed method, the LFE can be directly applied on the filtered k-space raw data, without any need for phase image reconstruction and subsequent spatial Fourier transform. One must also remain aware that the filtered k-space is convolved by the magnitude spectrum,as seen in Eq. [14]. Although this influence is deemed to be 
negligible in most cases, high frequencies corresponding to strong magnitude variations may add noise to the filtered k-space and influence the elastogram. Further work is needed to investigate such artifacts on the resulting elastogram. This may be particularly important in situations with significant physiological motion, resulting in residual, potentially periodic patterns that are not due to MRE shear wave motion. In such cases, motion correction methods may be necessary.

As we have already mentioned, a qualitative wave pattern image can be obtained without prior phase image reconstruction (Eq. [17]). However, the wave pattern image returned by the proposed method is weighted by different parameters, such as the magnitude image, the mechanical wave amplitude and the parameters of motion encoding. Therefore, such signal is not directly exploitable at this point beyond the sole qualitative visualization of the wave field, and it cannot be compared to the usual displacement image in conventional MRE used for further processing and other inverse solving methods. The relationship between the signal described by Eq. [17] and the actual displacement, as well as its sensitivity to noise compared to the one in conventional MRE need to be thoroughly studied to evaluate whether this method could be coupled with other inverse problem solving methods and post-processing techniques.

Substantial developments are still needed to make this method compatible with multi-channel arrays and undersampled k-space raw data. However, this approach could lead to new applications and alternative ways of estimating biomechanical properties in the future. Ongoing work is focused on in vivo monitoring of thermal ablations using this method for elastogram reconstruction in real time.

\section{Conclusion}

An alternative method for MRE data processing is proposed here. Elastograms can be independently computed without prior phase image reconstruction. Despite its limitations regarding the minimum number of phase offsets and the influence of the magnitude on the elastogram, this method is particularly well suited for applications requiring fast update rate of MRE information and it provides an interesting alternative to the conventional MRE process. 


\section{Acknowledgements}

This work was partly funded by the French state funds managed by the ANR (within the Investissements d'Avenir programme for the Labex CAMI); Grant number: ANR-11-LABX-0004, and the IHU Strasbourg; Grant number: ANR-10-IAHU-02.

\section{Compliance with Ethical standards}

Conflict of Interest: The authors declare no conflict of interest.

Research involving Animals: All applicable international, national, and/or institutional guidelines for the care and use of animals were followed. In vivo experiments of this study were performed according a protocol approved by a local ethics committee (ICOMETH C2EA - 38).

Informed consent: Not applicable to this study.

\section{Author's contribution}

Corbin: Protocol \& Project development; Data collection or management; Data Analysis

Breton: Protocol \& Project development; Data collection or management

De Mathelin: Protocol \& Project development;

Vappou: Protocol \& Project development; Data collection or management

\section{Appendix}

A temporal Fourier transform is applied on a set of k-space raw data acquired with varying delays between mechanical excitation and motion encoding gradients. By selecting only the temporal frequency of interest, most of the harmonics of the spatial frequency are removed.

The result is described by Eq. [12]: 


$$
\begin{aligned}
& S\left(v_{x}, 1\right) \\
& =\sum_{n \in U} \sum_{x=0}^{N_{x}-1} M(x) e^{j \phi_{0}(x)} e^{-\frac{j 2 \pi\left(v_{x} x\right)}{N_{x}}} j^{n} N_{t} J_{n}\left(C_{1}(x)\right) e^{j n\left(-\frac{\left(k_{x} x\right)}{N_{x}}+C_{2}\right)} \\
& U=\left\{n \mid n=k N_{t}+1, k \in Z\right\}
\end{aligned}
$$

As described by the set $U$, the component of interest $(n=1)$ is not the only one component selected by the filtering because of aliasing. In most cases, the other components are negligible compared to the first one (blue signal in Fig. 8.b), nevertheless the selection of other non-negligible components remains possible when the number of phase-offsets is poorly chosen as illustrated in Fig. 8.c-d (red signal). It is therefore relevant to assess conditions that favor an optimal ratio between the amplitude of the $1^{\text {st }}$ and the $\left(k N_{t}+1\right)^{\text {th }}, \mathrm{k} \in Z^{*}$ components of the spectrum that are susceptible to being associated to the same temporal frequency. The ratio $R$ is deduced from the previous equation:

$$
R=\log \left(\frac{J_{1}\left(C_{1}\right)}{J_{k N+1}\left(C_{1}\right)}\right)
$$

Let us consider that a component is negligible compared to the first one when its amplitude is at least 10 times inferior, i.e. when $R>1$. The number of phase-offsets $N_{t}$ required to satisfy this condition is plotted in Fig. 9 with respect to the $C_{1}$ parameter. Under the assumption that $R>1$, Eq.[12] simplifies to Eq.[14]

$$
S\left(v_{x}, 1\right)=\sum_{x=0}^{N_{x}-1} M(x) e^{j \phi_{0}(x)} e^{-\frac{j 2 \pi\left(v_{x} x\right)}{N_{x}}} j N_{t} J_{1}\left(C_{1}(x)\right) e^{j\left(-\frac{k_{x} x}{N_{x}}+C_{2}\right)}
$$



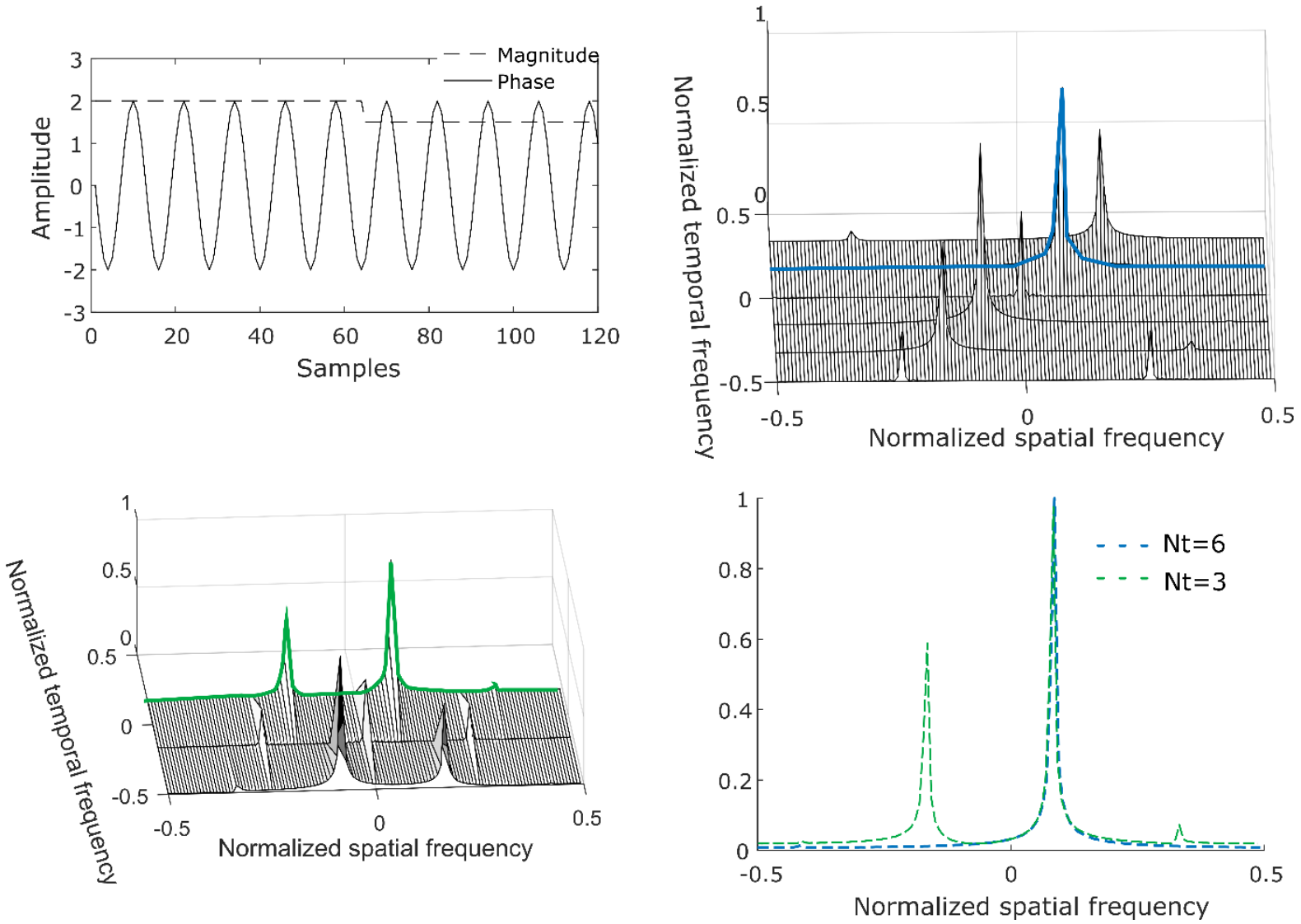

Fig. 8 a) Simulated 1D MR signal with heterogeneous magnitude and sinusoidal phase. b) Plot of the temporal Fourier transform of the previous k-space performed from the data set simulating 6 varying delays between motion encoding gradients and mechanical excitation. c) Plot of the temporal Fourier transform performed from the data set simulating 3 varying delays between motion encoding gradients and mechanical excitation. d) Plot of the filtering at the temporal frequency of interest in blue for the case with 6 phase-offsets and in red for the case with 3 phase-offsets 


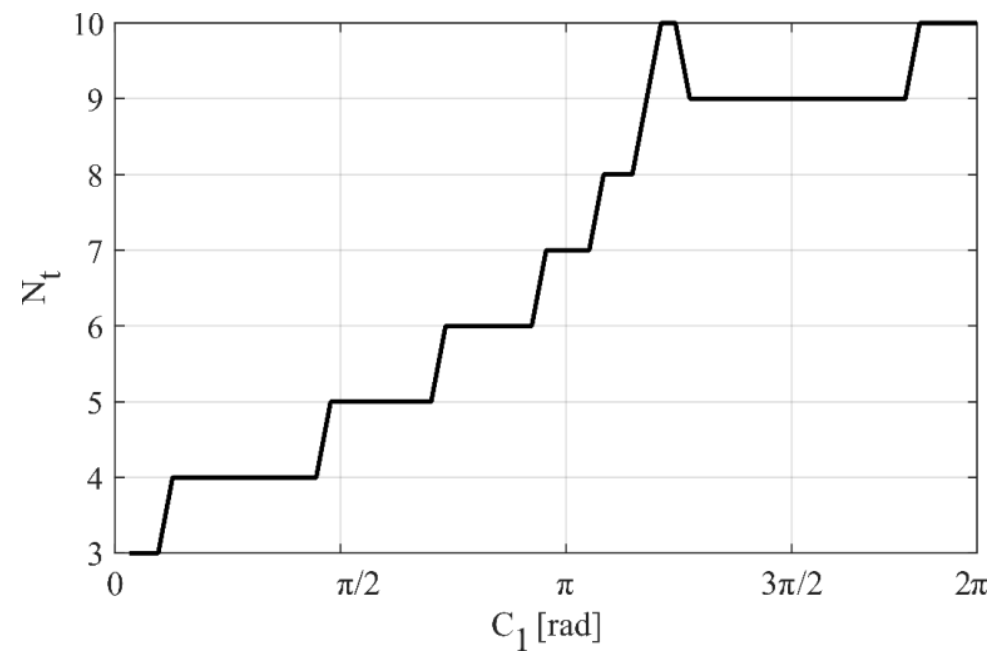

Fig. 9 Assessment of the minimal number of phase-offsets $N_{t}$ required to satisfy the condition R $>1$ with respect to the amplitude of the encoded wave $C_{1}$

\section{References}

1. Asbach P, Klatt D, Schlosser B, Biermer M, Muche M, Rieger A, Loddenkemper C, Somasundaram R, Berg T, Hamm B, Braun J, Sack I (2010) Viscoelasticity-based Staging of Hepatic Fibrosis with Multifrequency MR Elastography1. Radiology 257:80-86.

2. Huwart L, Peeters F, Sinkus R, Annet L, Salameh N, ter Beek LC, Horsmans Y, Van Beers BE (2006) Liver fibrosis: non-invasive assessment with MR elastography. NMR Biomed 19:173-179.

3. Garteiser P, Doblas S, Daire J-L, Wagner M, Leitao H, Vilgrain V, Sinkus R, Van Beers BE (2012) MR elastography of liver tumours: value of viscoelastic properties for tumour characterisation. Eur Radiol 22:2169-2177.

4. Rouvière O, Yin M, Dresner MA, Rossman PJ, Burgart LJ, Fidler JL, Ehman RL (2006) MR elastography of the liver: Preliminary results. Radiology 240:440-448. 
5. Sinkus R, Lorenzen J, Schrader D, Lorenzen M, Dargatz M, Holz D (2000) High-resolution tensor MR elastography for breast tumour detection. Phys Med Biol 45:1649.

6. Murphy MC, Curran GL, Glaser KJ, Rossman PJ, Huston J, Poduslo JF, Jack CR, Felmlee JP, Ehman RL (2012) Magnetic resonance elastography of the brain in a mouse model of Alzheimer's disease: initial results. Magn Reson Imaging 30:535-539.

7. Schregel K, Wuerfel née Tysiak E, Garteiser P, Gemeinhardt I, Prozorovski T, Aktas O, Merz H, Petersen D, Wuerfel J, Sinkus R (2012) Demyelination reduces brain parenchymal stiffness quantified in vivo by magnetic resonance elastography. Proc Natl Acad Sci USA 109:66506655.

8. Streitberger K-J, Sack I, Krefting D, Pfüller C, Braun J, Paul F, Wuerfel J (2012) Brain Viscoelasticity Alteration in Chronic-Progressive Multiple Sclerosis. PLOS ONE 7:e29888.

9. Muthupillai R, Rossman PJ, Lomas DJ, Greenleaf JF, Riederer SJ, Ehman RL (1996) Magnetic resonance imaging of transverse acoustic strain waves. Magn Reson Med 36:266-274.

10. Braun J, Guo J, Lützkendorf R, Stadler J, Papazoglou S, Hirsch S, Sack I, Bernarding J (2014) Highresolution mechanical imaging of the human brain by three-dimensional multifrequency magnetic resonance elastography at 7T. Neurolmage 90:308-314.

11. Klatt D, Hamhaber U, Asbach P, Braun J, Sack I (2007) Noninvasive assessment of the rheological behavior of human organs using multifrequency MR elastography: A study of brain and liver viscoelasticity. Phys Med Biol 52:7281-7294.

12. Papazoglou S, Hirsch S, Braun J, Sack I (2012) Multifrequency inversion in magnetic resonance elastography. Phys Med Biol 57:2329-2346. 
13. Perrinez PR, Kennedy FE, Van Houten EEW, Weaver JB, Paulsen KD (2010) Magnetic resonance poroelastography: An algorithm for estimating the mechanical properties of fluid-saturated soft tissues. IEEE Trans Med Imaging 29:746-755.

14. Vappou J, Breton E, Choquet P, Goetz C, Willinger R, Constantinesco A (2007) Magnetic resonance elastography compared with rotational rheometry for in vitro brain tissue viscoelasticity measurement. Magn Reson Mater Phy 20:273-278.

15. Chen J, Woodrum DA, Glaser KJ, Murphy MC, Gorny K, Ehman R (2013) Assessment of in vivo laser ablation using MR elastography with an inertial driver. Magn Reson Med 72:59-67.

16. Corbin N, Vappou J, Breton E, Boehler Q, Barbé L, Renaud P, de Mathelin M (2016) Interventional MR elastography for MRI-guided percutaneous procedures. Magn Reson Med 75:1110-1118.

17. Mariani A, Kwiecinski W, Pernot M, Balvay D, Tanter M, Clement O, Cuenod CA, Zinzindohoue F (2014) Real time shear waves elastography monitoring of thermal ablation: in vivo evaluation in pig livers. J Surg Res 188:37-43.

18. Corbin N, Vappou J, Rao P, Wach B, Barbé L, Renaud P, de Mathelin M, Breton E (2016) In vivo monitoring of percutaneous thermal ablation by simultaneous MR Elastography and Thermometry. In: Proceedings of the 24th scientific meeting, International Society for Magnetic Resonance in Medicine, Singapore, p 205

19. Rump J, Klatt D, Braun J, Warmuth C, Sack I (2007) Fractional encoding of harmonic motions in MR elastography. Magn Reson Med 57:388-395.

20. Garteiser P, Sahebjavaher RS, Ter Beek LC, Salcudean S, Vilgrain V, Van Beers BE, Sinkus R (2013) Rapid acquisition of multifrequency, multislice and multidirectional MR elastography data with a fractionally encoded gradient echo sequence. NMR Biomed 26:1326-1335. 
21. Murphy MC, Glaser KJ, Manduca A, Felmlee JP, Huston J, Ehman RL (2010) Analysis of time reduction methods for magnetic resonance elastography of the brain. Magn Reson Imaging 28:1514-1524.

22. Sack I, Beierbach B, Hamhaber U, Klatt D, Braun J (2008) Non-invasive measurement of brain viscoelasticity using magnetic resonance elastography. NMR Biomed 21:265-271.

23. Colton D, Kress R (1998) Inverse Acoustic and Electromagnetic Scattering Theory. Springer Science \& Business Media, New York, USA

24. Riek JK, Tekalp AM, Smith WE (1993) Effect of Z-motion in the phase of the K-space MRI data and identification of periodic Z-motion kernels. In: Proceedings of the IEEE International Conference on Acoustics, Speech, and Signal Processing, Minneapolis, USA, pp 511-514

25. Knutsson H, Westin C-F, Granlund G (1994) Local multiscale frequency and bandwidth estimation. In: Proceedings of the IEEE International Conference on Image Processing, Austin, USA, pp 3640

26. Manduca A, Muthupillai R, Rossman PJ, Greenleaf JF, Ehman RL (1996) Image analysis for magnetic resonance elastography. In: Proceedings of the IEEE International Conference on Engineering in Medicine and Biology Society, Amsterdam, The Netherlands, pp 756-757

27. Goldstein RM, Zebker HA, Werner CL (1988) Satellite radar interferometry: two-dimensional phase unwrapping. Radio Sci 23:713-720.

28. Barnhill E, Kennedy P, Johnson CL, Mada M, Roberts N (2014) Real-time 4D phase unwrapping applied to magnetic resonance elastography. Magn Reson Med 73:2321-2331.

29. Wang H, Weaver JB, Perreard II, Doyley MM, Paulsen KD (2011) A three-dimensional qualityguided phase unwrapping method for MR elastography. Phys Med Biol 56:3935-3952. 